\title{
Comparative study of human papilloma virus DNA detection and results of histopathological examination of cervical colposcopic biopsy
}

\author{
Ahmad M. Ragab Shalaby ${ }^{1}$ M.D., Ahmad Mostafa Shalaby ${ }^{2}$ M.D., Saed M. Naguib ${ }^{3}$ M.D. \\ 1 Department of Oncologic Pathology, Damanhour National Medical Institute, Damanhour, El-Behera, \\ Egypt. \\ 2 Department of Obstetric and Gynecology, Damanhour National Medical Institute, Damanhour, El- \\ Behera, Egypt. \\ 3 Department of Clinical Pathology and Immunology, Damanhour National Medical Institute, \\ Damanhour, El-Behera, Egypt.
}

Received: 1 November 2006; accepted: 12 July 2007

\begin{abstract}
Background: There is mounting evidence for HPV involvement in cervical cancer Human Papilloma Virus DNA is detected by hybridization techniques in $75-100 \%$ of patients with condylomas, precancerous cervical dysplasia, and invasive carcinoma.

Objective: The aim of this study was investigating factors that may contribute to falsenegative colposcopic biopsy results in positive high-risk HPV DNA results.

Material and Methods: Patients positive for high-risk human papillomavirus (HPV) DNA with negative cervical histopathologic findings were examined between January 2004 and August 2006.

Results: Patients with atypical squamous cells of undetermined significance (ASC) in Papanicolaou smears, with positive HPV DNA results, but negative cervical histopathologic findings accounted for $4.5 \%$ of all ASC smears submitted for HPV DNA testing. We found $4 \%$ of the cases had focal HPV infection or mild dysplasia. When serial sectioning of the biopsy material were examined, we found that $29 \%$ had clinically significant lesions: HPV infection or cervical intraepithelial neoplasia CIN 1, $18 \%$; CIN II/III, 8\%; and dysplasia, not otherwise specified (which we can not categorize into any group), $3 \%$. Of the remaining patients, follow-up revealed squamous abnormalities in $25 \%$. About $5 \%$ of patients with positive HPV DNA results had a negative follow-up biopsy result. "False-negative" biopsies accounted for one third of cases.
\end{abstract}

Conclusion: In almost one third of cases, clinically significant lesions were found when additional levels were examined.

Key words: Cervix, Dysplasia, Hybrid,Human papilloma virus.

\section{Introduction}

Epidemiologic data have long implicated a sexually transmitted agent, based specifically on the risk factors for cervical cancer. These risk

\section{Correspondence Author:}

Dr. Ahmad M. Ragab Shalaby, Department of Oncologic Pathology, Damanhour National Medical Institute, Damanhour, El-Behera, Egypt.

E-mail: ahmad_shalaby20@hotmail.com factors include early age at first intercourse, multiple sexual partners, and a male partner with multiple previous sexual partners. A recent survey of gynecologic cytology reporting practices found a median reporting rate of $3.9 \%$ for atypical squamous cells of undetermined significance (ASC) (1). Although in most patients with ASC a significant lesion will not be found in subsequent examination, they require further evaluation because $5 \%$ to $10 \%$ of patients initially diagnosed with ASC actually have high-grade dysplasia (2). 
Until recently, management of patients with ASC often involved colposcopic examination and cervical biopsy (3).

Human Papilloma Virus (HPV) is the known cause of the venereally transmitted vulvar condyloma accuminatum. It is also suspected to be an oncogenic agent in a variety of squamous tumors and proliferative lesions of skin and mucus membranes; however, there is mounting evidence for HPV involvement in cervical cancer $(4,5)$. The strongest evidence is that HPV DNA is detected by hybridization techniques in $75-100 \%$ of patients with condylomas, precancerous cervical dysplasia, and invasive carcinoma. Since the mid to late 1990s, testing for HPV DNA in Pap tests has been shown to be a useful adjunct in triaging patients with ASC test results for colposcopy. It has been shown that HPV DNA testing using the Hybrid Capture II assay, or HCII (Digene, Beltsville, $\mathrm{MD}$ ), performed better than repeated cytology in triaging patients with ASC (4). In addition, reflex high-risk HPV DNA testing offers the same life expectancy while remaining more cost-effective than other management strategies $(5,6)$.

In a recent meta-analysis, it has been reported that in a small but significant number of women, no abnormalities were found by colposcopicdirected cervical biopsy after a diagnosis of ASC with positive high-risk HPV DNA testing (4). In most cytologic-histologic correlation studies, colposcopic biopsy often is regarded as the "gold standard" on which gynecologic cytologic screening is to be judged. However, several studies have demonstrated that histologic examination is far from perfect. The aim of this study was investigating factors that may contribute to falsenegative colposcopic biopsy results in positive high-risk HPV DNA results.

\section{Materials and methods}

The material of this study included all recorded cases in the archive of the Medical Records Department of the Institute received in our lab from different Governorate areas and referred to Damanhour National Medical Institute Hospital (DNMI) - Egypt, during the period from January 2004 to August 2006.

A computerized search identified patients with ASC Pap test results and positive results of reflexive high-risk HPV DNA testing during the period from January 2004 to August 2006. All Pap tests included in the study were liquid-based preparations. The medical records were reviewed. Patients who underwent subsequent colposcopic- directed biopsy and/or endocervical curettage with no histologic evidence of HPV infection or dysplasia were selected for the study.

Cytologic specimens were obtained by using an endocervical brush. Reflex HPV DNA testing was performed on the residual sample after the cytologic report was issued and within 2 weeks of receipt of the specimen by the laboratory.

HPV DNA testing was performed using the Hybrid Capture II System according to the manufacturer's protocol. Briefly, double-stranded DNA is denatured into single-stranded DNA and combined with RNA probes. If HPV DNA is present in the specimen, the resulting RNA-DNA hybrids are immobilized in a capture tube coated with antibodies that recognize and bind the hybrids. A second, enzyme-linked antibody, when added to the capture tube, is capable of binding the immobilized RNA-DNA hybrids at multiple sites. On the addition of a chemiluminescent substrate, the presence of HPV DNA is detected. The presence of multiple binding sites for the detection antibody provides a means for signal amplification. Only high-risk HPV types $(16,18,31,33)$ were tested in accordance with the ASCCP guidelines (6). A threshold of $1 \mathrm{pg}$ of HPV DNA per milliliter of test solution was considered a positive result.

All surgical specimens were fixed in $10 \%$ buffered formalin. Then 3- to 4-mm blocks were made, processed routinely, and embedded in paraffin blocks. Three levels were obtained from each block and stained with H\&E.

The original H\&E-stained glass slides were reviewed. For cases that still were diagnosed as negative for dysplasia or HPV cytopathic effect on review, 3 additional H\&E-stained levels were obtained. For the few cases in which there was disagreement regarding the original and reviewed diagnoses, the discrepancy was recorded, and no other tissue sections were ordered. The diagnoses made on the new tissue levels were compared with the original histologic diagnoses.

\section{Statistical analysis}

Results are expressed as the mean. Clinical and other data were analyzed using Mann-Whitney, as applied by the computerized statistical program SPSS.

\section{Results}

From January 2004 to August 2006, a total of 1983 Pap tests were evaluated in our lab. Approximately $80 \%$ were ThinPrep preparations, and the remaining specimens were conventional 
smears. Of all the Pap tests reviewed, 240 (12.1\%) were diagnosed as ASC. Only cases submitted in liquid-based preparation were eligible for high-risk HPV DNA testing. As a result, 126 ASC (Figure 1, 2) Pap tests were submitted for HPV DNA testing. In 14 cases $(10.5 \%)$, the quantity was insufficient and the specimens were excluded from the study, leaving a total of 112 cases that ultimately were evaluated. High-risk HPV DNA was detected in 38 cases, accounting for $34.0 \%$ of all cases with sufficient quantity for HPV DNA testing.

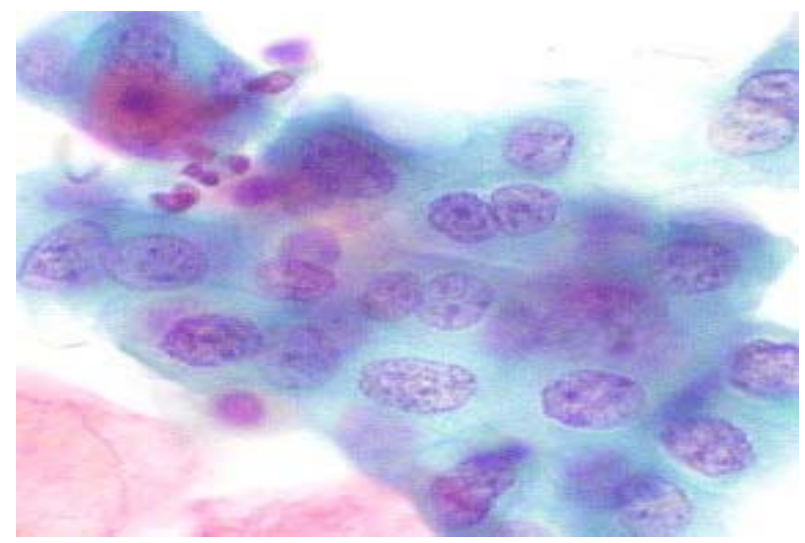

Figure 1. Atypical squamous cells (ASC) Thin Prep x 400

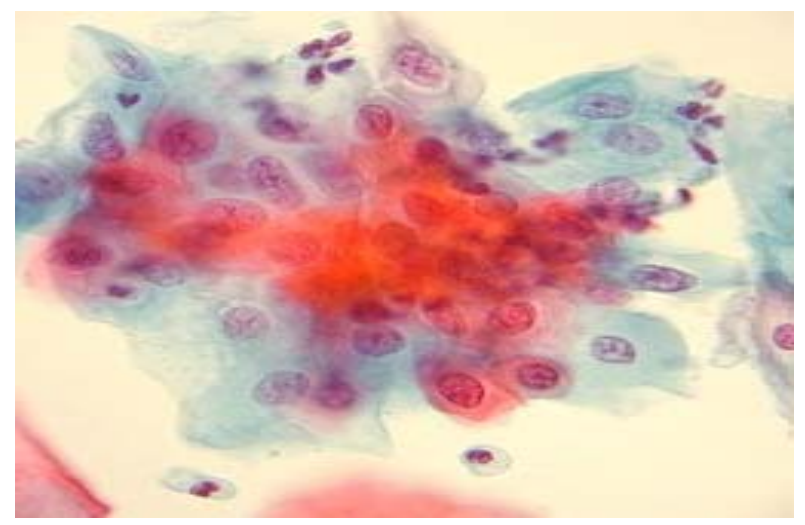

Figure 2. Atypical squamous cells (ASC) Thin Prep. X 200

Overall, $28.0 \%$ of patients with an ASC interpretation underwent histologic follow-up. Of these patients, $19(62.1 \%)$ had high-risk HPV DNA identified by Hybrid Capture, and of these, $17(87.8 \%)$ of the patients had a cervical intraepithelial neoplasia (CIN) lesion (Figure 3). As a result, $12.2 \%$ of the cases were identified with positive reflexive HPV DNA testing for high-risk subtypes and a negative cervical biopsy result, accounting for $4.5 \%$ of all ASC Pap tests submitted for reflexive HPV DNA testing. Paraffin blocks were not available for 1 case, which was excluded. The biopsy specimens were obtained within 1 year from the collection of the cytology specimens; the median interval between the biopsy and the Pap test was 6 months.

Review of the original H\&E-stained sections revealed that 4 cases had histologic evidence of HPV or mild cervical dysplasia (CIN 1). Moderate and severe dysplasias were not observed. In the remaining cases, no histologic evidence of dysplasia or HPV cytopathic changes was noted during review. When 3 additional H\&E-stained levels were reviewed, clinically significant lesions were noted in $29 \%$ of the cases.

HPV or low-grade dysplasia (CIN 1) was noted in $18 \%$ of cases, high-grade dysplasia (CIN 2/3) in $8 \%$ of cases, and dysplasia, not otherwise specified, in 3\% of cases (Table I) (Figure 3, $4 \mathrm{~A}$, B).

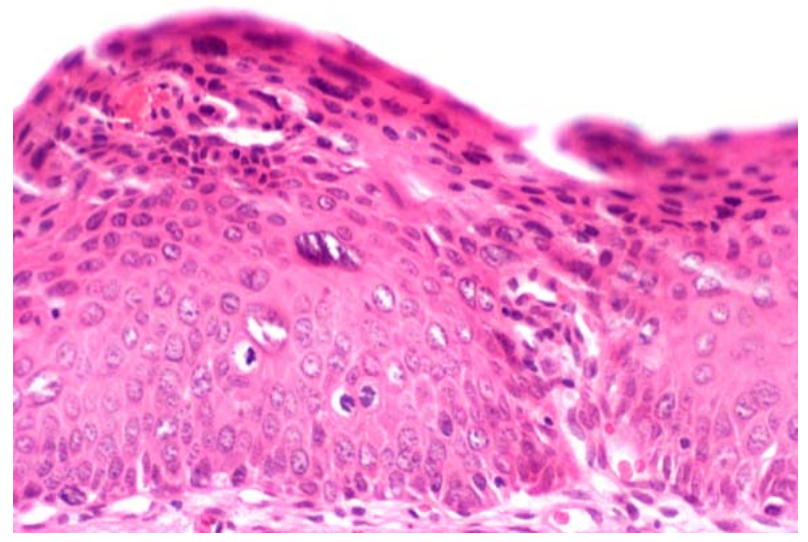

Figure 3. Moderate cervical dysplasia CIN II (H\&Ex 200)
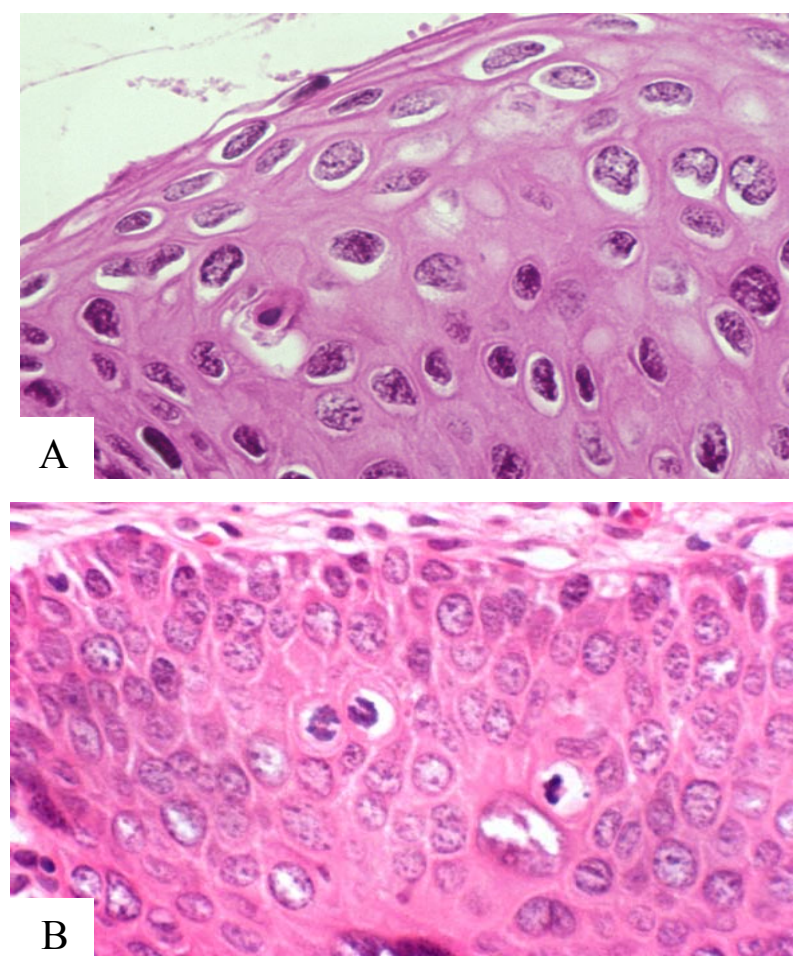

Figure 4. Severe Cervical Dysplasia with HPV-16 infection (H \& E x 400) 
In total of $29 \%$ of patients were lost in follow-up. Among patients who had been followed-up, 14\% had persistent ASC Pap test results. In addition, $11 \%$ patients had cytologic and/or histologic evidence of clinically significant lesions on followup, $9 \%$ had low-grade dysplasia or HPV cytopathic changes, and $2 \%$ had high-grade dysplasia. The remaining patients had negative repeated Pap test results.

Table I. Distribution of clinically significant findings on review of original sections and additional levels in the studied cases*

\begin{tabular}{lcc}
\hline Findings" & First Review & Second Review \\
\hline HPV/ low-grade dysplasia & 4 & 18 \\
High-grade dysplasia & 0 & 8 \\
Dysplasia, NOS & 0 & 3 \\
Total & 4 & 29
\end{tabular}

HPV: human papillomavirus, NOS: not otherwise specified.

*Data are given as percentage.

\section{Discussion}

Many authors agree with statement that correlation of cytologic and corresponding histologic findings is one of the several recommended performance indicators in gynecologic cytology (9-14).

The reported rates of cytologic-histologic differences range from $11 \%$ to $47 \%(8,14-18)$. According to the College of American Pathologists' Q-Probes study of 22,439 correlations in 348 laboratories, the mean rate of cytologichistologic discrepancies was $16.5 \%(11,32)$. Because of the medicolegal implications, it is not surprising that most cytologic-histologic correlative studies have focused on false-negative cytologic diagnoses. In contrast, relatively little has been written on the subject of false-positive cytologic diagnoses (16-18). The reported rate of false-positive cases ranged from $5.5 \%$ to $7.0 \%$ $(7,18)$. Tritz et al $(1995)$, reported that factors related to colposcopic biopsy, including sampling and interpretation errors, were the most common causes of a discrepancy between cytologic and corresponding histologic findings $(8,31)$. Also, Anderson and Jones (1997) demonstrated that as many as $45 \%$ of the cases subsequently developed SIL, indicating an initial "false-negative" biopsy result rather than a "false-positive" cytologic result in a review of negative cervical biopsy specimens with corresponding cytologic specimens positive for a squamous intraepithelial lesion (SIL) during the preceding 3 months (7). Therefore, in our study, rather than accepting histologic findings as the true indicator of cervical pathology, we attempted to determine the extent of histologicrelated factors that contribute to false-positive high-risk HPV DNA results.

Many factors have been found to influence the outcome of cytologic-histologic correlative studies. These factors include the quality of cytology specimen collection, fixation, processing, and interpretation. The outcome of the correlative study also depends on the quality of colposcopic examination, biopsy specimen collection, biopsy specimen processing, the extent of biopsy specimen evaluation, ie, the number of sections and levels examined, and the biopsy diagnosis.

ASCCP published new guidelines for managing patients with cervical cytologic abnormalities $(6,33)$. It is recommended that patients with an initial cytologic interpretation of ASC be tested for high-risk HPV DNA. A recent meta-analysis reported that the sensitivity and specificity of HPV DNA testing for detection of high-grade cervical dysplasia were $94.8 \%$ and $67.3 \%$, respectively (4).These authors also reported a substantial number of false-positive cases, ranging from $16 \%$ to $45 \%$. In our study, we found a false-positive rate of $4.5 \%$, much lower than the results reported in the literature. Our end point was the detection of biopsy-proven CIN 1 or HPV cytopathic changes or worse, whereas for the meta-analysis, biopsyconfirmed CIN 2 or worse was the end point. Coste et al (2003) reported a false-positive rate of $6.2 \%$ for HPV DNA testing when used in the setting of cervical cancer screening (19). Our study population consisted of patients who were diagnosed with ASC, whereas the populations of other cytologic-histologic correlation studies before the use of HPV DNA testing consisted of patients with a cytologic diagnosis of low-grade SIL or worse $(7,34)$. Even so, our false-positive rate was compatible with the rates in these studies.

Most studies agree with our results in use of histologic findings rather than cytologic findings as the gold standard. Ferris et al (2005) for the ASCUS-LSIL Triage study (ALTS) trial reported poor inter-observer agreement $(\kappa=0.36)$ in colposcopy $(20,34)$. In our study, about one third of the "false-positive" cases could be attributed to histologic examination-related factors. Underinterpretation of the biopsy specimen is one such factor. Inter-observer variability in evaluating cervical biopsy specimens has been reported (21, 24). The differentiation between reactive squamous cellular changes and low-grade SILs (CIN 1 and HPV cytopathic changes) is particularly difficult 
and controversial histologically. About $4 \%$ of our false-positive cases were due to underinterpretation of HPV cytopathic changes or CIN 1 in the biopsy specimen. We did not encounter any cases in which a high-grade SIL (CIN II/III) was under-diagnosed. Although it could be argued that this $4 \%$ error rate merely represents inter-observer variability, our rate is similar to that reported in the literature, where errors in interpretation account for $3.4 \%$ to $33 \%$ of all cases with false-positive cytologic-histologic discrepancie $(8,17)$.

Histopathological factors, including the number of levels examined have been found to be the major source of errors that could account for some false-positive HPV DNA results. In our study, we found HPV cytopathic changes and/or dysplasia in approximately one third of the cervical biopsy specimens when additional levels were obtained. Many workers agree with these observations (8, 17).

Another possible explanation for false-positive HPV DNA testing is found in the follow-up data for patients who tested positive for high-risk HPV but had negative biopsy results despite examination of additional histologic levels. Of this subset of patients, $11 \%$ showed an SIL in subsequent studies within a 1-year period after the initial negative biopsy result. An additional 14\% continued to have abnormal cells on Pap tests. The initial negative biopsy result may be due to sampling error during colposcopic examination. This is supported by the ALTS trial that showed that the 2-year cumulative risk of developing CIN 2 or 3 was $27 \%$ in women with HPV-positive ASCUS and an initially negative colposcopic biopsy result $(25,26)$.

Although high-risk HPV DNA testing is more objective than the Pap test, legitimate falsepositive HPV DNA test results have been encountered (19). One situation that may give rise to false-positive results is when the previous sample contains a high load of low-risk HPV DNA; chemiluminescent emission from such a sample might result in false reactions in contiguous samples. Although the specificity of the second generation of the Hybrid Capture assay is quite good, the presence of a very high load of low-risk HPV DNA in the sample can cross-hybridize with high-risk HPV DNA probes, giving rise to falsepositive results for the high-risk subtypes. The incidence is very low, less than $2 \%$, and is dependent on the cutoff value $(19,22,23)$. However, cross-hybridization with low-risk HPV DNA should not result in a discrepancy between HPV DNA test and histologic results.
There were a substantial number of ASC cases in the present study population with a positive HPV DNA test result, a negative biopsy result, and a negative repeated Pap test result, supporting the validity of the negative biopsy diagnosis. This may be due to the natural history of HPV infection. It has been reported that HPV infections can be transient and episodic, especially in younger women $(27,28)$. If the HPV infection for a particular woman is newly acquired, the infection most likely will resolve; the longer an infection persists, the less likely this becomes. According to Ho et al (1998) the probability that a newly acquired HPV infection will resolve is $31 \%$ during the first 6-month period, 39\% during the second and $11 \%$ in the third 6-month period after infection (27). A similar pattern also is observed with SILs $(29,30)$. Most of our cases underwent biopsy within one year of Pap or HPV DNA test. It is reasonable to assume that some of the newly acquired HPV infections might have resolved before the patients underwent colposcopic examination and directed biopsy, resulting in falsepositive HPV DNA testing.

\section{Conclusion}

In almost one third of cases, clinically significant lesions were found when additional levels were examined. Regression of HPV infection and the associated morphologic abnormalities provide a likely explanation for another percentage of the discrepant cases. We recommend that additional levels have to be obtained when initial histologic sections do not demonstrate evidence of dysplasia or HPV cytopathic changes after a cytologic diagnosis of ASC and a positive HPV DNA test result. This is particularly important if there is a discrepancy between the size of the tissue sample on the slide and that observed grossly. Close follow-up is crucial when the initial cervical colposcopic biopsy result is negative because a small number of patients will have squamous abnormalities in subsequent examination.

\section{Acknowledgements}

We thank Professor Nayera Anwar AbdAlHameid and Professor Nadia Mahmoud Mokhtar (Professors of Oncologic Pathology in National Cancer Institute, Cairo University) for their great help and advise that make this work possible. 


\section{References}

1. Neal MH, Wilbur DC, Davey DD. Bethesda 2001 implementation and reporting rates: 2003 practices of participants in the College of American Pathologists Interlaboratory Comparison Program in Cervicovaginal Cytology. Arch Pathol Lab Med 2004;128:1224-1229.

2. Kinney WK, Hurley LB, Manos MM. Identifying women with cervical neoplasia: using human papillomavirus DNA testing for equivocal Papanicolaou results. JAMA 1999;281:1605-1610.

3. ASCUS-LSIL Triage Study (ALTS) Group. Results of a randomized trial on the management of cytology interpretations of atypical squamous cells of undetermined significance. Am J Obstet Gynecol 2003;188:1383-1392.

4. Buntinx F, Van Ranst M, Arbyn M. Virologic versus cytologic triage of women with equivocal Pap smears: a meta-analysis of the accuracy to detect high-grade intraepithelial neoplasia. J Natl Cancer Inst 2004;96:280293.

5. Wright TC, Goldie SJ. Cost-effectiveness of alternative triage strategies for atypical squamous cells of undetermined significance. JAMA 2002;287:2382-2390.

6. Wright TC Jr, Cox JT, Massad LS. 2001 Consensus Guidelines for the management of women with cervical cytological abnormalities. JAMA 2002;287:2120-2129.

7. Anderson MB, Jones BA. False positive cervicovaginal cytology: a follow-up study. Acta Cytol 1997;41:16971700.

8. Tritz DM, Weeks JA, Spires SE. Etiologies for noncorrelating cervical cytologies and biopsies. Am J Clin Pathol 1995;103:594-597.

9. Herrero R. Sexual behavior, Venereal diseases, Hygiene practices and invasive cervical cancer in a high risk population. Cancer 1990;65:380-386.

10. CLIA. Cytology: Section 493.1274. 2005. Available at: http://www.phppo.cdc.gov/clia/regs/subpart_k.aspx\#493. 1274. Accessed June 28, 2005.

11. Jones BA, Novis DA. Cervical biopsy-cytology correlation: a College of American Pathologists Q-Probes study of 22439 correlations in 348 laboratories. Arch Pathol Lab Med 1996;120:523-531.

12. Koss LG. The Papanicolaou test for cervical cancer detection; a triumph and a tragedy. JAMA 1989;261:737743.

13. Kraemer BB. Quality assurance activities of the College of American Pathologists. Acta Cytol 1989;33:434-438.

14. Rohr LR. Quality assurance in gynecologic cytology: what is practical? Am J Clin Pathol 1990;94:754-758.

15. DiBonito L, Falconieri G, Tomasic G. Cervical cytopathology: an evaluation of its accuracy based on cytohistologic comparison. Cancer 1993;72:3002-3006.

16. Dodd LG, Sneige N, Villarreal Y. Quality-assurance study of simultaneously sampled, non-correlating cervical cytology and biopsies. Diagn Cytopathol 1993;9:138-144.

17. Crum CP, Cibas ES. Cytologic/histologic correlation for quality control in cervicovaginal cytology: experience with 1,582 paired cases. Am J Clin Pathol 1995;103:3234.

18. McCord ML, Stovall TG, Summitt RL Jr. Discrepancy of cervical cytology and colposcopic biopsy: is cervical conization necessary? Obstet Gynecol 1991;77:715-719.

19. Coste J, Sastre-Garau X, de Cremoux P. Efficiency of the Hybrid Capture 2 HPV DNA test in cervical cancer screening: a study by the French Society of Clinical Cytology. Am J Clin Pathol 2003;120:492-499.

20. Ferris DG, Litaker M. Interobserver agreement for colposcopy quality control using digitized colposcopic images during the ALTS trial. J Low Genit Tract Dis 2005;9:29-35.

21. Ismail SM, Colclough AB, Dinnen JS. Observer variation in histopathological diagnosis and grading of cervical intraepithelial neoplasia. BMJ 1989;298:707-710.

22. Klinkhamer PJ, Vooijs GP, de Haan AF. Intraobserver and interobserver variability in the diagnosis of epithelial abnormalities in cervical smears. Acta Cytol 1988;32:794-800.

23. Parker MF, Zahn CM, Vogel KM. Discrepancy in the interpretation of cervical histology by gynecologic pathologists. Obstet Gynecol 2002;100:277-280.

24. Stoler MH, Schiffman M; Atypical Squamous Cells of Undetermined Significance-Low-Grade Squamous Intraepithelial Lesion Triage Study (ALTS) Group. Interobserver reproducibility of cervical cytologic and histologic interpretations: realistic estimates from the ASCUS-LSIL Triage Study. JAMA 2001;285:15001505.

25. Schiffman M, Solomon D. Prospective follow-up suggests similar risk of subsequent cervical intraepithelial neoplasia grade 2 or 3 among women with cervical intraepithelial neoplasia grade 1 or negative colposcopy and directed biopsy. Am J Obstet Gynecol 2003; $188: 1406-1412$.

26. Schiffman M, Solomon D, Guido R. Postcolposcopy management strategies for women referred with lowgrade squamous intraepithelial lesions or human papillomavirus DNA-positive atypical squamous cells of undetermined significance: a two-year prospective study. Am J Obstet Gynecol 2003;188:1401-1405.

27. Ho GY, Bierman R, Beardsley L. Natural history of cervicovaginal papillomavirus infection in young women. N Engl J Med 1998;338:423-428.

28. Collins S, Winter H, Woodman CB. Natural history of cervical human papillomavirus infection in young women: a longitudinal cohort study. Lancet 2001;357:1831-1836.

29. Nasiell K, Nasiell M, Vaclavinkova V. Behavior of moderate cervical dysplasia during long-term follow-up. Obstet Gynecol 1983;61:609-614.

30. Richart RM, Barron BA. A follow-up study of patients with cervical dysplasia. Am J Obstet Gynecol 1969;105:386-393.

31. Koutsky LA, Ault KA, Wheeler CM; Proof of Principle Study Investigators. A controlled trial of a human papillomavirus type 16 vaccine. N Engl J Med 2002;347:1645-1651.

32. Harper DM, Franco EL, Wheeler C. For the GlaxoSmithKline HPV Vaccine Study Group. Efficacy of a bivalent $\mathrm{L} 1$ virus-like particle vaccine in prevention of infection with human papillomavirus types 16 and 18 in young women: a randomised controlled trial. Lancet 2004;364:1757-1765.

33. Villa LL, Costa RL, Petta CA. Prophylactic quadrivalent human papillomavirus (types 6, 11, 16, and 18) L1 viruslike particle vaccine in young women: a randomised double-blind placebo-controlled multicentre phase II efficacy trial. Lancet Oncol 2005;6:271-278.

34. Mao C, Koutsky LA, Ault KA. Efficacy of human papillomavirus-16 vaccine to prevent cervical intraepithelial neoplasia: a randomized controlled trial. Obstet Gynecol 2006;107:18-27. 